\title{
LA ENSEÑANZA EN EL LABORATORIO. ¿QUÉ PODEMOS APRENDER EN TÉRMINOS DE CONOCIMIENTO PRÁCTICO Y DE ACTITUDES HACIA LA CIENCIA?
}

\author{
SÉRÉ, MARIE-GENEVIÈVE \\ DidaScO, Université Paris Sud XI. F-91405 Orsay
}

\begin{abstract}
Resumen. Se trata aquí de destacar aquellas cosas irreemplazables que la actividad experimental aporta a la enseñanza en relación con otros métodos. Para el estudiante, cuando realiza trabajos prácticos, se trata de «comprender» y de «aprender», pero también de algo muy diferente, de «hacer» y de «aprender a hacer». A fin de explorar las ideas clásicas de objetivos conceptuales, procedimentales y epistemológicos, mostramos cómo la teoría puede ponerse al servicio de la práctica, mientras que lo contrario es actualmente lo más frecuente. Los trabajos prácticos son una excelente forma de aprender las teorías de las ciencias físicas.Mostraremos que, al estar los conocimientos procedimentales al servicio de la práctica, la experimentación es la ocasión para adquirirlos. Por último, al ser aprendidos al mismo tiempo que una visión construida de la ciencia, permiten iniciativa y autonomía a los estudiantes. El conjunto de trabajos sintetizado aquí invita a revisar los objetivos de los trabajos prácticos en el bachillerato y en el primer ciclo de la universidad.
\end{abstract}

Palabras clave. Trabajos prácticos, contenidos conceptuales, contenidos procedimentales, epistemología.

Summary. In this paper, the irreplaceable things that experimental activity introduces in teaching compared to other methodologies are highlighted. For students, practical work is about «understanding» and «learning», but also about something very different, «doing» and «learning how to do». In order to explore the classical notions of conceptual, procedural and epistemological goals, it is shown how theory can serve practice, while the opposite is currently more frequent. Practical work is an excellent way to learn physical theories.It is shown that, when procedures serve practice, experimental work is the occasion to acquire them. Lastly, when these contents are learnt along with a constructed view on science, they permit students initiative and autonomy. Previous research summarised here invites to review the goals of practical work in high school and college.

Keywords. Practical work, contents, procedures, nature of science.

\section{INTRODUCCIÓN}

Entre las actividades a disposición del profesor para que los estudiantes aprendan ciencias, hay algunas que son particularmente complejas: son aquéllas que implican la experimentación. Su eficacia muchas veces es puesta en duda (N'Tombela, 1998). Es por ello que resulta todavía necesario reflexionar sobre la mejor manera de utilizarlas y situarlas en las perspectivas actuales de la enseñanza de las ciencias. La experimentación puede intervenir en la enseñanza siguiendo diferentes modalidades. Se examinarán aquí más específicamente las sesiones durante las cuales los estudiantes participan en la realización de experimentos, lo que se llama trabajos prácticos (TP). Se trata de identificar aquello irreemplazable que aportan los trabajos prácticos en relación con otros métodos que ya han mostrado ser eficaces o que se están difundiendo (las tecnologías de la información y la comunicación). Este trabajo intenta actualizar los tesoros todavía ocultos de los trabajos prácticos e investigar cómo «la experiencia puede servir más de una sola vez», contrariamente a lo que dice el proverbio.

De una manera esquemática, se puede decir que se esperan diferentes tipos de resultados de la enseñanza de las ciencias en su conjunto. Estos resultados se pueden detallar de la siguiente manera:

- comprender la teoría, es decir, los conceptos, los modelos, las leyes, los razonamientos específicos, que muy a menudo difieren notablemente de los razonamientos corrientes; 
- aprender toda esta teoría;

- realizar experiencias mostrando un cierto número de realidades, hechos y aparatos que utilizan teorías y procedimientos, para adquirir la experiencia, en el sentido que se da a este término en inglés;

- aprender a rehacer las mismas experiencias con los mismos procedimientos;

- aprender los procedimientos y los caminos para poder utilizarlos cuando se trate de realizar otras experiencias en otros contextos;

- aprender a usar el saber teórico aprendido para que esté presente y sea utilizado cuando se trate de realizar un proceso completo de investigación.

Como se puede ver, en esta lista encontramos «comprender»y «aprender», operaciones esencialmente intelectuales. También encontramos «realizar», «aprender a hacer», que se sitúan del lado de la acción y de la realización y, por tanto, implican decisiones, juicios e iniciativas. Es necesario introducir eficazmente en los TP este aprendizaje del «hacer».

En el esquema de la figura 1, «hacer» y «comprender» tienen una parte común. Intentaremos mostrar que los
TP ayudan a comprender y que el aprendizaje conceptual ayuda a hacer ciencias. El aprendizaje se sitúa tanto dentro del «comprender» como del «hacer»; sin embargo, por la importancia de la evaluación, los estudiantes y profesores se sienten tentados a otorgar una gran importancia al aprendizaje de conceptos, modelos y razonamiento. Sin embargo, si se pretende hacer auténticamente ciencias, la apuesta es aprovechar las ventajas evidentes, examinadas y conocidas, de los trabajos prácticos, a saber:

- la motivación que los trabajos prácticos aportan a los estudiantes (Lunetta y Tamir, 1979);

- el interés de razonar sobre lo concreto del caso particular del experimento, más que sobre lo abstracto en las clases de aula y en las sesiones habituales de ejercicios;

- el interés de visualizar los objetos y eventos que la ciencia conceptualiza y explica.

Algunos filósofos han expresado cómo el pensamiento es más completo cuando es capaz de juicio y toma en cuenta la dimensión de la acción. Así, Bergson pregona «pensar como un hombre de acción, actuar como un hombre de pensamiento» (citado por Renaud-Coulon y Sérieyx, 1999). En cuanto a Piaget, él es sensible a la dimensión realizadora del pensamiento, que tiene en

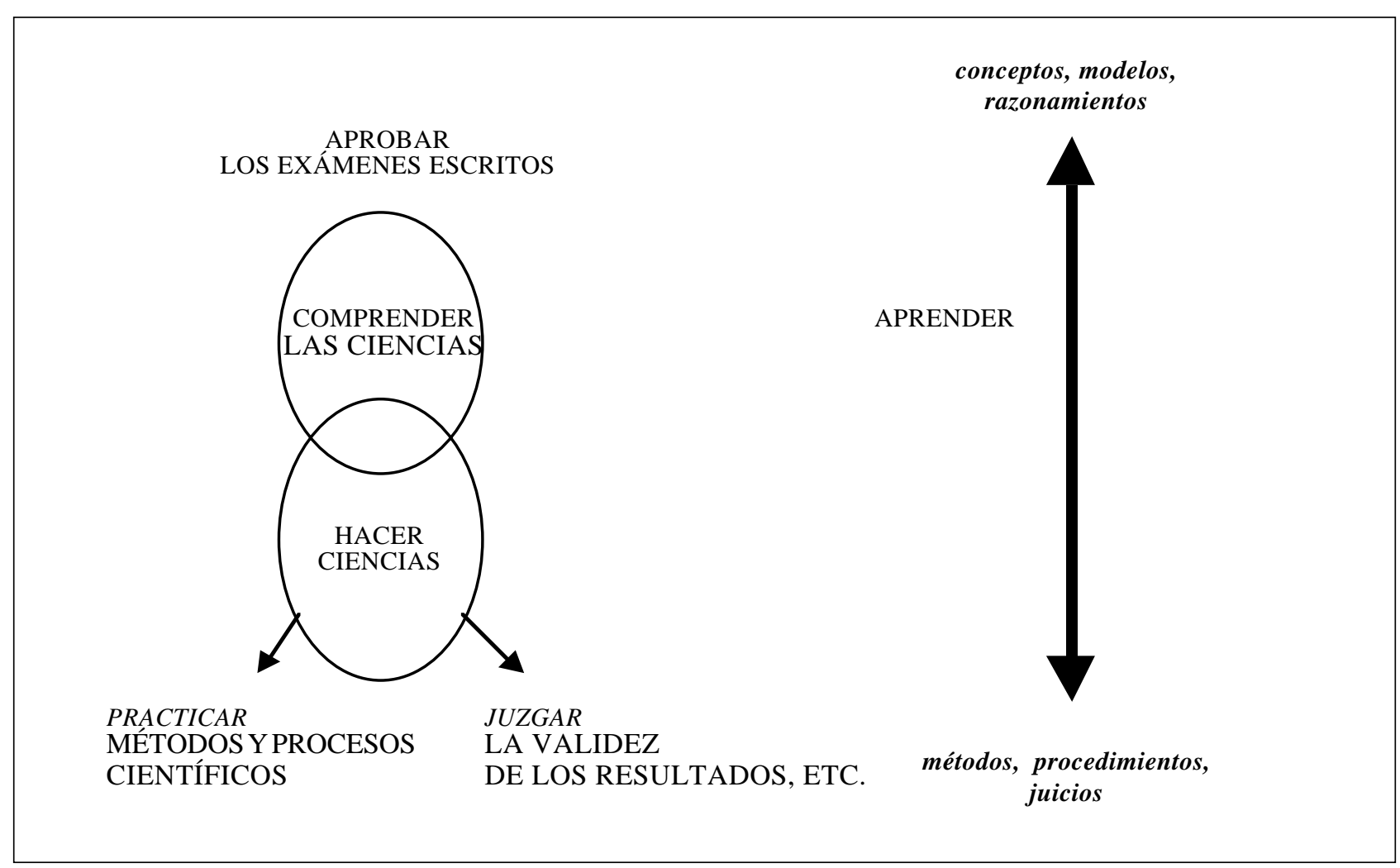


cuenta especialmente en un libro con un título significativo: Conseguir (réussir) y comprender (1972).

Para iluminar lo que los TP aportan de original a la enseñanza de las ciencias, hace falta reflexionar de nuevo sobre los objetivos que permiten alcanzar. Sin presuponer una separación neta entre estos diferentes tipos de objetivos, se pueden examinar sucesivamente los tres tipos clásicos, para revisarlos a la luz de los trabajos actuales sobre la enseñanza y del mejor conocimiento epistemológico de las ciencias de que disponemos. Examinaremos, pues, sucesivamente los objetivos conceptuales, procedimentales y epistemológicos a la luz de trabajos recientes, en particular aquéllos del proyecto europeo de investigación en didáctica (19961998) que ha reunido a investigadores de siete países (Séré, 1998): «Improving science education: issues and research on innovative empirical and computer-based approaches to labwork in Europe» (título corto: «Labwork in science education»).

En esta exploración de las posibilidades de los TP en términos del aprendizaje, se abordarán las siguientes cuestiones:

- ¿Pueden los conocimientos teóricos ser puestos al servicio de la práctica experimental?

- ¿Cómo están los conocimientos procedimentales al servicio de la práctica?

- ¿Qué rol asignar a las actitudes hacia la ciencia para que estén al servicio de la actividad práctica?

\section{¿PUEDEN LOS CONOCIMIENTOS TEÓRI- COS SER PUESTOS AL SERVICIO DE LA PRÁCTICA EXPERIMENTAL?}

Podemos preguntarnos acerca de la efectividad de los TP para alcanzar objetivos conceptuales, ya que éstos son específicos de otros métodos de enseñanza (clases teóricas y resolución de problemas). Es cierto que los profesores frecuentemente preparan experiencias en las cuales el aprendizaje de lo conceptual pasa por lo práctico, donde la práctica está al servicio de la teoría (Hucke y Fischer, 1998). Es el caso de numerosos TP que consisten en verificar una ley o en encontrar una que ya es perfectamente conocida desde antes. Los investigadores describen entonces el pasaje «del mundo de los objetos al mundo de las ideas». Muestran que este pasaje se realiza a menudo bastante mal. Los estudiantes, que desean ante todo realizar correctamente la tarea práctica, encuentran procedimientos para evitar las nociones teóricas (Beney, 1998; Haller et al., en prensa).

Sin embargo, existen TP en los cuales, al contrario, la teoría está al servicio de la práctica. En efecto, pueden encontrarse manipulaciones en las cuales, para actuar, los estudiantes se ven forzados de alguna manera a utilizar los conceptos. Éste es el caso, por ejemplo, de todas las veces que el «mundo de los objetos» no se distingue completamente del «mundo de las ideas». En las manipulaciones propuestas en el bachillerato, se encuentran muchos objetos que no pueden ser utilizados y comprendidos más que teniendo ya algunas «ideas» en la cabeza. Se encuentran fácilmente ejemplos en electricidad (la máquina de Whimshurst, la jaula de Faraday), pero también en otras áreas. En el caso de estos aparatos, los estudiantes no pueden evolucionar en el «mundo del objeto» si no son capaces de evolucionar en el «mundo de las ideas». Por el contrario, una piel de gato y un destornillador «tester» hecho de un pequeño tubo de neón, que permiten estudiar los mismos conceptos, muestran claramente que el mundo de los objetos y el de las ideas están más nítidamente separados.

Jenkins (1979) califica este tipo de aparatos de «intellectually constructed things» (cosas construidas intelectualmente). También podemos llamar así a numerosos instrumentos de medición. Hacking (1983) los llama «theory made objects» (objetos hechos de teoría).

Entendiendo y manipulando aparatos «cargados de teoría», los estudiantes pueden utilizar numerosas nociones teóricas, más que verificarlas. Los órdenes de magnitud elegidos, la disposición del aparato, los sistemas que mejor aseguran la reproducibilidad, son todos conocimientos prácticos que a los estudiantes les interesa guardar en la memoria para luego utilizar (Guillon y Séré, en prensa). Por ello, la primera fase de los TP no debería ser descuidada. La identificación de los aparatos, así como las elecciones hechas por quienes concibieron la experiencia, deberían ser revisadas, estudiadas y justificadas. Aquí el conocimiento teórico no es puesto en cuestión. Es utilizado y produce un conocimiento práctico que puede ser almacenado en situaciones en las cuales los estudiantes deben organizarse por sí mismos.

Aunque los objetivos conceptuales son los que más frecuentemente buscan los TP, todavía queda por imaginar cuestionamientos y guías para que los conocimientos conceptuales contribuyan a fabricar reales conocimientos prácticos. Los dos tipos de conocimientos pueden así instalarse juntos y eficazmente en la cabeza de los estudiantes.

\section{LOS CONOCIMIENTOS PROCEDIMENTA- LES AL SERVICIO DE LA PRÁCTICA}

Los conocimientos procedimentales son por esencia saberes prácticos, en el sentido de que permiten la concepción de los experimentos y racionalizan la acción. Están muy presentes cuando se conciben los trabajos prácticos escolares, pero raramente son tenidos en cuenta por los estudiantes. Existe una gran variedad de procedimientos; la situación es ligeramente diferente en las diversas disciplinas. En biología y en química, la importancia de los procedimientos está más reconocida e incluso da lugar a evaluaciones (Coquidé-Cantor y Desbeaux-Salviat, 2001). Tomaremos aquí como ejemplo una sesión de trabajos prácticos de física para mostrar que tal actividad didáctica encubre numerosos pro- 
cedimientos, a menudo escondidos, pero que, sin embargo, tienen sentido y son reutilizables por los estudiantes en otros contextos. En efecto, aparece la cuestión de saber si los procedimientos dan lugar a conocimientos generalizables o si son específicos de un contexto y de una situación. Hace falta también preguntarse si es suficiente utilizar un procedimiento en un contex to dado para conocerlo y reutilizarlo o, incluso, si es posible enseñarlo formalmente.

Quien concibió la sesión del ejemplo de más abajo ha utilizado numerosos procedimientos. Hemos observado a los estudiantes en funcionamiento en la clase en el aula (Séré y Beney, en prensa): tenían una guía, que les recordaba los pequeños trozos de teoría indispensables y les explicaba lo que tenían que hacer. Y hacían todo lo más rápidamente posible siguiendo cuidadosamente la guía de las tareas. Respetando estas condiciones de trabajo del todo habituales, hicimos a los estudiantes unas preguntas escritas inmediatamente antes de los TP. Precisamos los resultados de estas preguntas mediante algunas entrevistas, con la intención de saber lo que se había aprendido.

\section{Un ejemplo: la medición de la velocidad de la luz.}

El fenómeno de propagación de la luz da lugar a un montaje simple, que se muestra en la parte derecha de la figura 2. La introducción del procedimiento de medición (parte izquierda de la figura) suscita muchos problemas complementarios, cuyas soluciones deberían aportar nuevos conocimientos a los estudiantes.

Con el objetivo de entender todo lo que los estudiantes podrían, en principio, adquirir a partir de esta sesión, hemos hecho una lista de los procedimientos que quien la concibió puso en práctica para que la medición fuera posible. Luego hemos mirado lo que los estudiantes retuvieron al realizar el trabajo que se les pedía. Damos aquí algunos elementos de este análisis (Beney, 1998).

\section{Adaptación de una señal al instrumento de medición utilizado}

Se trata aquí de adaptar la frecuencia de la señal luminosa a la gama de frecuencias admitidas por el osciloscopio. El método consiste en utilizar modulaciones sucesivas de las diferentes señales.

Señal luminosa: Se utilizan componentes electrónicos que no son suficientemente rápidos para reaccionar a la frecuencia de la luz del día. Entonces se modula la señal para obtener una frecuencia mucho más baja que la de la luz visible; esto permite trabajar bajo la luz del día sin que la señal recibida sea parasitada por la luz ambiente. Este procedimiento no cambia las propiedades de la onda progresiva. Se puede así trabajar con una intensidad cuya variación temporal es compatible con las curvas de respuesta del diodo receptor.

Señal eléctrica: Se utiliza la posibilidad de transformar una señal eléctrica de gran frecuencia en una señal de frecuencia más pequeña, «mezclándola» con otra señal de frecuencia vecina. Se utiliza también un filtrado de las señales eléctricas.

Estas transformaciones permiten adaptar las señales a las capacidades de los instrumentos de medición.

He aquí lo que dice un estudiante durante una entrevista individual que sigue a la manipulación:

«Ponemos el rayo luminoso en forma de onda. De esta forma, le damos una especie de cronología poniéndolo en forma de onda y después, eh... medimos la diferencia de fase entre la onda tal como parte y la onda tal como llega.»

Está claro que el procedimiento está confuso en la cabeza de este estudiante.

Figura 2

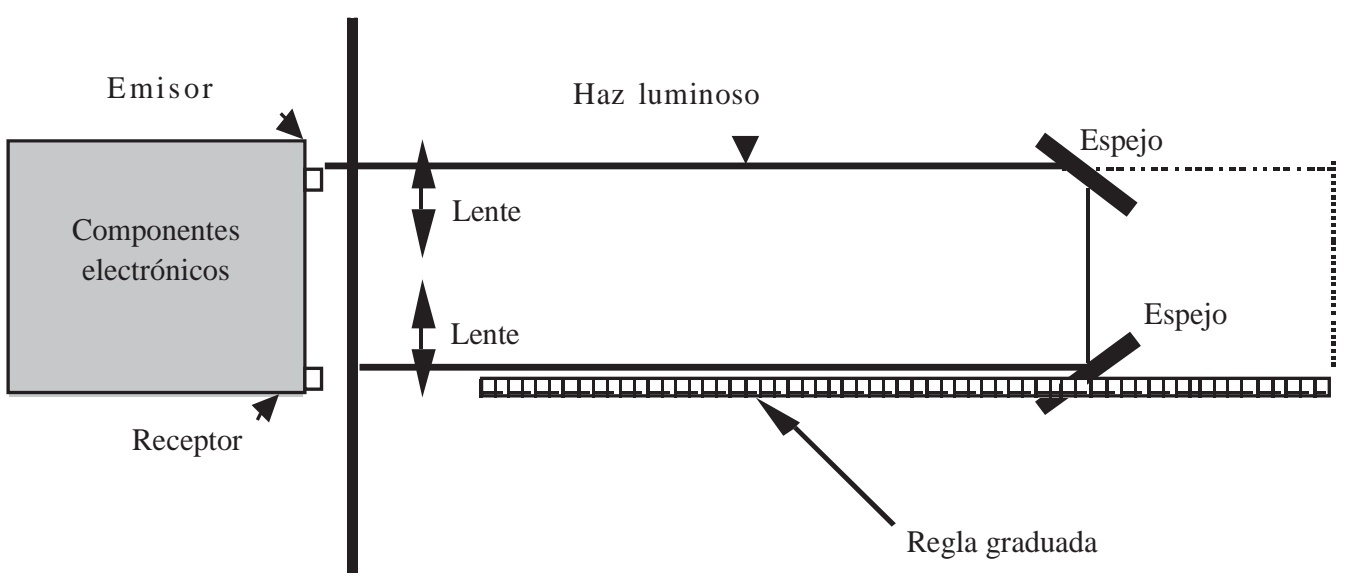


También hemos formulado por escrito algunas preguntas más fáciles, fundamentales, con respecto a este procedimiento de adaptación de la señal a los instrumentos de medición. Mostramos aquí debajo las preguntas y los porcentajes de respuestas correctas para unos sesenta estudiantes:

El osciloscopio que acabas de utilizar, ¿permite estudiar señales de cualquier frecuencia? (69\%)

¿Cuál es la gama de frecuencias utilizable con este osciloscopio? (54\%)

La frecuencia de la señal luminosa, ¿pertenece a esta gama? (69\%)

Da el orden de magnitud de la frecuencia de la señal luminosa que has utilizado. (12\%)

Se ve que más de un tercio de los estudiantes no tomó conciencia del interés del método.

\section{Medición por comparación cuando no se tiene acceso al cero de la cantidad a medir}

Aquí se trata de medir la distancia recorrida por la luz por diferencia entre dos posiciones. El método permite efectuar mediciones exactas y consiste en medir el tiempo de recorrido de la luz entre dos posiciones de los espejos.

Hemos planteado la siguiente pregunta por escrito:

Durante la medición de la velocidad de la luz en el agua, el cero de la regla graduada no coincide con la cara de entrada del tubo lleno de agua. ¿Provoca este hecho que el resultado sea solamente aproximado?

El resultado puede ser exacto. (63\%)

Medición por comparación con el valor conocido de una magnitud cuando el instrumento de medición no es el más apropiado

La pregunta escrita es la siguiente:

Un osciloscopio permite medir tiempos a partir de la fase de las señales que permite visualizar. En tu opinión, los valores de tiempo obtenidos:

- Son sólo órdenes de magnitud. (33\%)

Explícalo.

- Permiten sólo comparaciones. (38\%)

Explícalo.

- Son absolutos (se pueden tener en cuenta los valores obtenidos independientemente de toda comparación). (31\%) Respuesta correcta

- En este caso, menciona algunas de las condiciones a cumplir para obtener medidas de tiempo absolutas. (28\%) Respuesta correcta
A partir de estas dos preguntas, se ve que alrededor de dos tercios de los estudiantes entienden el procedimiento de medición por diferencia, y que menos de un tercio comprenden el procedimiento de medición por comparación.

\section{Calibración de un instrumento de medición por com- paración con una referencia}

Al ser la precisión de un osciloscopio limitada, hizo falta verificar la calibración de la base de tiempos. Este procedimiento requiere la utilización de una señal de referencia y del botón de calibración.

\section{Optimización de la precisión de una medida}

- Eligiendo un valor particular o una zona de valores.

- Eligiendo el calibre para minimizar el error de la lectura en la medición de los tiempos.

\section{Elección del tipo de tratamiento de las mediciones}

El texto que guía a los estudiantes impone el tipo de tratamiento de datos, como es a menudo el caso en este tipo de TP estándar. Los tratamientos de datos impuestos son los siguientes:

1) Medición de $c$ en el aire: efectuar una medida. Evaluar su precisión.

2) Medición de $c$ en el agua. Efectuar 5 medidas. Calcular la media y calcular $\sigma$ (desviación cuadrática media).

3) Medición de $c$ en un medio transparente. Trazar la curva $\Delta \mathrm{d}=\mathrm{f}(\Delta \mathrm{t})$. De ella deducir $c$.

Todo esto provee muchas posibilidades de reflexión sobre los diferentes métodos utilizados. Hemos constatado que los estudiantes no realizan esta reflexión.

En el transcurso del mismo semestre, los estudiantes tenían la ocasión de ver muchos otros procedimientos frecuentemente utilizados en física: regulación, medición de una magnitud independiente del tiempo por medio de un fenómeno dependiente del tiempo, identificación de varios fenómenos que contribuyen a la misma señal para poner sólo uno en evidencia, etc. Nuestras observaciones han mostrado que la conciencia que tenían los estudiantes de haber utilizado estos procedimientos era muy pobre. Sin embargo, estos procedimientos permiten medir y observar más y mejor. Y esto no es específico de la física. El vínculo estrecho entre procedimientos y observación ha sido establecido por numerosos autores en diferentes formas. Francis Bacon (1561-1626) era el precursor de esta idea cuando señalaba que no es suficiente observar al león para conocerlo. Hace falta además «torcerle la cola al león».

Los procedimientos al servicio de la autonomía de los estudiantes

Este ejemplo conduce a pensar que los estudiantes se quedan intelectualmente pasivos cuando no entienden 
los procedimientos en juego. Estos procedimientos son indispensables para «torcer la cola del león». Siendo las virtudes de la autonomía y de la creatividad cada vez más reconocidas en la enseñanza, es necesario tender a poner a disposición de los estudiantes procedimientos como otras tantas herramientas. En Alemania se llevaron a cabo entrevistas abiertas a propósito de esta famosa motivación que se busca a través de los TP (Woolnough y Allsop, 1985). A propósito de las sesiones estándares de TP, similares a la del ejemplo de más arriba, los estudiantes se expresan de la siguiente manera:

«Preferiría investigar mis propias preguntas y no cosas que están tan predeterminadas.»

«[Durante el trabajo de laboratorio] lo que realmente echo en falta es que no hay posibilidad de construir el experimento en mi propia forma.»

El ejemplo anterior concernía a la física. Todos estos procedimientos de optimización de las mediciones se aplican igualmente en biología y en química. En ellas, es muy importante aprender la utilización de los materiales y los elementos de vidrio. Pero haría falta de vez en cuando ir más allá y esforzarse en dejar que los estudiantes elijan el nivel de observación en función del problema planteado: organismo, órgano, célula, molécula. Las limitaciones debidas a la variabilidad de los seres vivientes o a la irreversibilidad de las experiencias, deberían ser dejadas al juicio de los estudiantes o, al menos, debidamente discutidas (Coquidé et al., 1999). La organización de una experiencia testigo y el muestreo son procedimientos a menudo utilizados en biología y que deberían ser comprendidos por sí mismos.

Hace falta insistir, sin embargo, sobre el hecho de que el aprendizaje de procedimientos no es pertinente más que a propósito de un contexto experimental. Hemos visto que «hacer» no es suficiente para «aprender». Aun así, es indispensable «hacer»y tomar conciencia de lo que se hace, para «aprender» procedimientos, saber usarlos y llegar así a ser más autónomo en la experimentación.

\section{LAS ACTITUDES HACIA LA CIENCIA AL SERVICIO DE LA ACTIVIDAD PRÁCTICA}

En nuestros días, diferentes trabajos de investigación y diferentes innovaciones en la enseñanza de las ciencias convergen hacia la siguiente idea general: entender lo que es la ciencia o lo que son las ciencias forma parte del aprendizaje de las ciencias (Désautels y Larochelle, 1998). Ya existen revisiones de trabajos sobre este tema (Leach et al., 2000). Una definición posible de las actitudes hacia la ciencia es la siguiente (Doña y Salinas, 2001):

«Predisposición por la cual se tiende a reaccionar favorable o desfavorablemente hacia un objeto que puede ser una cosa, otra persona o una institución.»

Velazco y Salinas (2001) definen además las concepciones epistemológicas como:
«Comprensión de la naturaleza epistemológica de conceptos, leyes, teorías y modelos.»

La cuestión aquí es estudiar la relación entre la experimentación y la formación del juicio de los estudiantes sobre lo que es la ciencia; dicho en otros términos, investigar estas actitudes y concepciones epistemológicas durante las experiencias. Esto implica renunciar a hacer que los estudiantes sostengan un discurso coherente sobre «la» ciencia. Al contrario, nuestro trabajo consistió en hacerles comentar situaciones experimentales, para llegar a la imagen de ciencia que las tareas escolares de experimentación les sugieren. Para ello, recogimos dos tipos de datos: observaciones directas realizadas en el laboratorio, en Francia esencialmente, y preguntas escritas propuestas a los estudiantes en una encuesta realizada en Francia y en España con estudiantes de bachillerato y de primer curso de universidad (undergraduate level). El análisis completo de esta encuesta, organizada en el marco del proyecto europeo citado más arriba, está publicado en otra parte (Séré et al., 2001).

\section{Las reacciones de los estudiantes frente a resultados sorprendentes durante una experimentación}

Hemos formulado una pregunta escrita sobre el siguiente tema: durante unos TP de biología, ciertos grupos de estudiantes no obtienen los resultados esperados de acuerdo con lo que el profesor desea enseñar (el texto de la pregunta se adjunta en el anexo). Para una parte de los cuestionarios, hemos hecho una pregunta abierta (la respuesta debe ser una frase): ¿Qué debe hacer el profesor frente a aquellos resultados experimentales que contradicen la teoría que él desea presentar a sus estudiantes?

Luego, para el resto de los cuestionarios, con el fin de facilitar su explotación, hemos propuesto respuestas que los estudiantes debían marcar (se presenta el texto en el anexo). Esta segunda serie de respuestas arroja resultados similares, aunque menos explícitos que las frases de los estudiantes.

Las respuestas a las preguntas «abiertas» pueden ser colocadas en dos conjuntos de categorías:

- Los estudiantes proponen hacer otro test, recomenzar $\mathrm{o}$, menos frecuentemente, formular hipótesis sobre las razones del problema. Tenemos, por ejemplo, las siguientes respuestas:

«La realidad no siempre sigue a la teoría.»

«Los resultados a menudo son estadísticos en biología.»

- Hay menos estudiantes que piden que se les deje la iniciativa $(6 \%$ en el bachillerato y $10 \%$ en el primer curso de la universidad):

«Lo más importante es que los estudiantes sepan lo que es verdadero.» 
Mediante la evocación de una «verdad» científica, de una distancia inevitable entre la teoría y el experimento, vemos dibujarse la imagen que los estudiantes tienen en la cabeza. Por otra parte, algunos profesores son conscientes de que los TP provocan cierto escepticismo frente a la experiencia, y se preocupan de preservar a los estudiantes.

«Queremos la solución para prepararnos para el examen.»

«El profesor debe comenzar de nuevo mostrando lo que se ha de encontrar y diciendo lo que se ha de saber.»

Hace falta completar este resultado considerando las respuestas de profesores (franceses y españoles) a la misma pregunta. Un $25 \%$ de ellos (sobre todo biólogos) desea dejar la iniciativa a los estudiantes. Ningún profesor de universidad lo pregona.

Sobre el terreno, en las aulas o en los laboratorios, se confirma el hecho de que el objetivo que se privilegia en el TP es el refuerzo del aprendizaje conceptual. En efecto, se da poca importancia a los pasos, métodos y procedimientos. Esto da como resultado una gran pasividad en los estudiantes, que no están motivados para comprender los métodos y las elecciones realizadas, y sobre todo para apropiárselos a fin de tener posteriormente la capacidad de utilizar esos métodos. También se nota un escepticismo real en los estudiantes, convencidos de que ellos mismos no pueden encontrar nada valioso. Nos acercamos aquí a la preocupación de los profesores de primaria, que cuentan entre sus objetivos el dar a los niños una fuerte percepción de sus capacidades personales. Nos hemos dado cuenta de que, en el bachillerato, muchos de los estudiantes han perdido esta percepción.

\section{Las reacciones de los estudiantes frente a la disper- sión de medidas}

Hemos formulado una pregunta escrita sobre el siguiente tema: en un laboratorio, dos grupos realizan mediciones y encuentran resultados diversos. (En el anexo se encuentra el texto completo de las diferentes preguntas que formulamos.) ¿Qué se ha de tomar finalmente como resultado? A modo de ejemplo, tomaremos un solo caso, en el cual las dos series de mediciones diversas son las siguientes:

$\left[\begin{array}{lllll}94,9 \mathrm{~g} & 96,6 \mathrm{~g} & 97,1 \mathrm{~g} & 98,1 \mathrm{~g} \quad 98,9 \mathrm{~g}\end{array}\right]$ y $\left[\begin{array}{lll}91,9 \mathrm{~g} & 96,5 \mathrm{~g}\end{array}\right.$ $97,5 \mathrm{~g} \quad 97,5 \mathrm{~g} \quad 102,2 \mathrm{~g}]$

Estas dos series tienen la misma media $(97,12$ g), número que no forma parte de los valores obtenidos. Uno de los valores ha sido encontrado dos veces y constituye la moda $(97,5 \mathrm{~g})$.

No se encuentra ninguna respuesta totalmente «correcta», es decir, que utiliza la estadística y, en particular, da la media acompañada de un intervalo de confianza.

Se distinguen tres grandes tendencias, que hemos nombrado de la siguiente forma en inglés:
- Data driven. Los datos son tomados en cuenta por ellos mismos. Esto se manifiesta por el hecho de que la media es considerada como un valor aproximado, o incluso que la moda es la mejor representante de una serie de medidas dispersas. También, para los estudiantes de esta categoría, no se debe tomar la media si no figura entre las mediciones.

- Theory-driven. Para los estudiantes de esta categoría, al contrario, la media es reconocida como el único valor preciso. También piensan que, cuantos más valores se tengan, más correcto será el resultado (cuando se obtiene así más precisión que exactitud). El tratamiento de los datos es considerado importante, además de consideraciones teóricas para corregir los valores obtenidos, incluso si esto lleva a modificar notablemente los resultados obtenidos.

- Relativism. Los estudiantes de esta categoría expresan su incapacidad de encontrar por sí mismos resultados correctos:

«De todas formas hay una separación entre la teoría y la realidad.»

«La física no es una ciencia exacta.»

«Haría falta tener un valor teórico / calculado / oficial.»

Reencontramos, con variadas expresiones, la idea de que los estudiantes otorgan gran valor a todo aquello que es «calculado» u obtenido a partir de una teoría o por científicos autorizados.

Resumidamente, menos del $10 \%$ de los estudiantes sueña con cuestionar el protocolo de la experiencia o la elección de los instrumentos de medición. Parece que, en los TP más usados en Francia como en el resto de Europa, las elecciones y las decisiones están fuera de las preocupaciones de los estudiantes.

Observaciones directas han permitido reencontrar estas diferentes actitudes, con una preponderancia del relativismo, así como la convicción de que es imposible decidir:

«Hay una única medida correcta.»

«Somos torpes. La dispersión de las medidas no existe.»

«Es nuestra culpa.»

Unas entrevistas más en profundidad muestran que la enseñanza de las ciencias tradicional ha producido en los estudiantes unas convicciones deterministas profundamente ancladas, aunque raramente conscientes, que los dejan impotentes frente a la variabilidad de la experiencia. Quedan entonces desprovistos para abordar el rol del azar y los formalismos probabilistas que se introducen actualmente en numerosos dominios de la ciencia (Séré, 1992). Parece urgente plantearse la cuestión del mejor momento para introducir la noción de azar en los currículos de ciencias. 
Las relaciones posibles entre teoría y experimentación, tal como las ven los estudiantes

En una pregunta escrita, los estudiantes debían elegir entre dos frases que se les presentaban. Además les pedíamos, y he aquí el interés de la pregunta, que justificaran su elección dando el ejemplo de biología, química, física, etc. que se les viniera a la mente.

Damos los resultados en porcentajes de estudiantes que han elegido cada una de las frases:

1) «Los científicos parten de conocimientos teóricos que influyen sobre su análisis de los datos.»

De acuerdo: $49 \%$ en el bachillerato y $30 \%$ en la universidad.

En desacuerdo: $39 \%$ en el bachillerato y $19 \%$ en la universidad.

El 12\% eligió la respuesta neutra: «No estoy seguro.»

2) Los científicos parten de los datos obtenidos, sin dejar que sus conocimientos teóricos interfieran con éstos.

De acuerdo: $22 \%$ en el bachillerato y $59 \%$ en la universidad

En desacuerdo: $11 \%$ en el bachillerato y $72 \%$ en la universidad.

El 20\% eligió la respuesta neutra: «No estoy seguro.»

Si se eliminan los estudiantes que no han querido pronunciarse, un estudiante dado puede dar cuatro pares de respuestas diferentes: de acuerdo con la primera frase y en desacuerdo con la segunda; la situación inversa, de acuerdo con las dos frases, en desacuerdo con las dos frases. El par de respuestas más frecuente, tanto en el bachillerato como en la universidad es: de acuerdo con «teoría antes» (1) y en desacuerdo con «datos ante todo» (2).

Habida cuenta de la generalidad de la pregunta, probablemente notada por los muchos estudiantes que no se pronunciaron, es importante considerar las situaciones que les vinieron a la mente para responder. Por orden decreciente del número de respuestas, encontramos que las situaciones evocadas son:

1) Situaciones de actividades experimentales realizadas en clase. Estas situaciones tienen efectivamente un impacto sobre el pensamiento de los estudiantes. Por ejemplo:

«Cuando aprendíamos a hacer dosificaciones, hacía falta conocer muchas reacciones de antemano.»

«Los TP de genética sobre los cruzamientos de maíz no son más que teoría.»

2) Situaciones históricas: algunos ejemplos han llamado visiblemente la atención de los estudiantes.
«Un científico debe tener intuición. Plutón fue descubierto de manera teórica.»

«Si los científicos partieran de conocimientos teóricos, todavía pensarían que la tierra no es redonda.»

Los científicos que son citados en apoyo de la frase que hemos resumido como «teoría antes» son Newton, Huygens, Einstein, Darwin, Charpak, Michelson y Morley. Aquéllos evocados para datos ante todo son Einstein, Le Verrier, Descartes y Bergson. Por último, cuando los estudiantes piensan que la respuesta depende del caso particular; citan a Bohr, Planck, Einstein, Koch, Galileo, Marie Curie y Lavoisier.

3) Algunos hechos provenientes del desarrollo actual de las disciplinas científicas:

«Los biólogos que estudian la genética han sido guiados por la teoría de Mendel y el descubrimiento del ADN.»

«El descubrimiento del bacilo de Koch y después el genio de la genética que investiga tal o cual gen muestran que los dos métodos son complementarios.»

En las aulas y en los laboratorios, se observa que los estudiantes tienen una débil conciencia de la variedad de los respectivos lugares de la teoría y la experiencia. Dada la importancia que los estudiantes han dado a las sesiones de TP en las situaciones que evocaron, parece que hace falta explotar esas sesiones en este sentido: los profesores deberían esforzarse por llevar a los estudiantes a una toma de conciencia de la variedad de relaciones entre teoría y experiencia en la experimentación.

Hace falta, entonces, tener en cuenta un resultado importante de estas diferentes encuestas: cada estudiante no tiene una posición epistemológica única, coherente entre una situación y otra (Leach et al., 2000). La formación de una imagen de las ciencias es compleja, por aproximaciones sucesivas y siguiendo una progresión que podemos calificar de constructivista.

En estas condiciones, ¿cómo llevar a los estudiantes a una visión de las ciencias utilizable en la práctica? ¿Cómo llevarlos hacia una actitud responsable hacia las ciencias?

Proponemos establecer los fundamentos de tal actitud sobre una sana visión de las relaciones entre teoría y experiencia, que puede ser formulada en términos de:

- la articulación entre las diferentes fases del trabajo del científico;

- la organización de la experimentación;

- los resultados que pueden esperarse de la observación y su dependencia de los instrumentos;

- la información que aportan las mediciones y los procedimientos para tratar esa información;

- el tipo de conclusiones que se pueden esperar. 
Nada de todo esto puede ser considerado intuitivo. Para llegar a ello, es pertinente que:

- sea una actividad guiada por los profesores en los TP;

- no se encierre a los estudiantes en una disciplina única;

- el trabajo epistemológico a partir de casos particulares sea interdisciplinario;

- no se busque enseñar una epistemología única;

- se busquen más bien tomas de conciencia epistemológica, no limitándose a cursos teóricos de filosofía de la ciencia. La complementariedad es posible.

\section{CONCLUSIÓN}

Los conocimientos acerca de los roles respectivos de la teoría y de la experiencia en la experimentación producen actitudes hacia la ciencia capaces de promover la iniciativa en la medida en que forman el juicio sobre los métodos. La distinción clásica entre objetivos conceptuales, procedimentales y epistemológicos no es muy utilizable. Todos aparecen muy unidos y dependientes los unos de los otros; allí estriba el interés, tanto como la dificultad, de los TP.

Esto es coherente con las ideas que Kuhn (1968) expresó con respecto a la enseñanza de las ciencias. Para él, los procedimientos y los métodos son los primeros elementos de la «matriz disciplinar», común a todos los expertos de una disciplina, y que se instala progresivamente en las mentes de los estudiantes durante sus estudios. Una vez adquiridos, procedimientos y métodos permiten resolver los problemas conceptuales que aparecen.

Este tipo de resultados aboga por que se reoriente el trabajo experimental sobre objetivos distintos de los objetivos conceptuales. Los TP pueden dar más cosas, y distintas, a los estudiantes.

Hace falta entonces poner al día los procedimientos en cada disciplina. Hace falta promover una reflexión sobre la naturaleza de la ciencia. El conjunto permite autonomía e iniciativa.

¿A qué tipo de público se dirige este tipo de enseñanza? Se insiste hoy en día acerca de la necesidad de que la naturaleza de la ciencia sea comprendida por todos, aquéllos que seguirán una carrera científica y los demás. En la vida cotidiana, las ciencias están cada vez más presentes y el nivel de los resultados mediatizados es cada vez más elevado. Los mismos científicos, cada vez más especializados, deben adquirir en el bachillerato visiones sobre las ciencias que no practicarán. En efecto, un físico nuclear está casi tan poco preparado frente a los avances actuales de la genética que un no-científico. Frente a la especialización, se debería introducir entre los estudiantes una imagen auténtica de las ciencias. Nuestra propuesta es utilizar los TP, disminuyendo su carga conceptual, en beneficio de la toma de conciencia de los procedimientos y elecciones epistemológicas, en términos de la relación entre la teoría y la experiencia.

Queda por hacer todo un trabajo de puesta a punto didáctica de los TP, que permita estas tomas de conciencia epistemológica y que provea a los estudiantes condiciones en las cuales ellos puedan ejercer elecciones y decisiones. El terreno es amplio en términos de innovaciones que utilicen la pedagogía de proyectos (a menudo asociada a la idea de la autonomía de los estudiantes), pero también en términos de reestructuración de los TP clásicos, buscando explotar mejor la autonomía latente que es posible, y guiando a los estudiantes hacia las preguntas que vale la pena formular.

Es tiempo de dejar atrás las dificultades actuales de la enseñanza, dando su lugar a los trabajos prácticos y esforzándose para que «la experiencia sirva más de una vez», contrariamente a lo que afirma el proverbio. La aportación de los TP puede ser irreemplazable para la educación de los científicos y de los ciudadanos.

\section{NOTA}

Ponencia presentada en el VI Congreso Internacional sobre Investigación en la Didáctica de las Ciencias (Barcelona, 12 al 15 de septiembre de 2001). Ha sido traducida del francés por Agustín Adúriz-Bravo. 


\section{REFERENCIAS BIBLIOGRÁFICAS}

BÉCU-ROBINAULT, K. (1998). Introduction of the power concept, en Psillos, D. y Niedderer, H. (eds.). Teaching and learning in the science laboratory. Dordrecht: Kluwer.

BENEY, M. (1998). «Contribution à l'analyse des phases manipulatoires des travaux pratiques de physique en premier cycle universitaire. Les apprentissages possibles à travers la conduite de l'action». Tesis presentada en la Université Paris Sud XI.

COQUIDÉ-CANTOR, M. y DESBEAUX-SALVIAT, B. (2001). Chimie et biologie: figures de rencontre. Didaskalia, 18, pp. 121-146.

COQUIDÉ-CANTOR, M., BOURGEOIS-VICTOR, P. y DESBEAUX-SALVIAT, B. (1999). Résistance du réel dans les pratiques expérimentales. Aster, 28, pp. 57-78.

DÉSAUTELS, J. y LAROCHELLE, M. (1998). The epistemology of students: the «thingified» nature of scientific knowledge, en Fraser, B. y Tobin, K. (eds.). International handbook of science education. Dordrecht: Kluwer.

DOÑA, M.E. y SALINAS, J. (2001). Dimensiones de un aprendizaje comprensivo de las ciencias: las actitudes científicas, en Retos de la enseñanza de las ciencias en el siglo XXI. VI Congreso Internacional sobre Investigación en la Didáctica de las Ciencias. Barcelona.

FERNÁNDEZ GONZÁLEZ, M., GONZÁLEZ GARCÍA, F., DE MANUEL, E., GALLEGOS, J.A., PERALES, F.J. y SÉRÉ, M.G. (en evaluación). Imágenes de la ciencia en relación con las prácticas experimentales.

GUILLON, A. y SÉRÉ, M.G. (1998). Open-ended projects accomplished by students during the second year of undergraduate studies, en Psillos, D. y Niedderer, H. (eds.). Teaching and learning in the science laboratory. Dordrecht: Kluwer.

HACKING, I. (1983). Representing and intervening. Cambridge: Cambridge University Press.

HALLER, K., WELZEL, M. y VON AUFSCHNAITER, S. (1998). Development of situated cognition during labwork activities of university students, en Psillos, D. y Niedderer, $\mathrm{H}$. (eds.). Teaching and learning in the science laboratory. Dordrecht: Kluwer.

HUCKE, L. y FISCHER, H.E. (1998). The link of theory and practice in traditional and in computer-based university laboratory experiments in Germany, en Psillos, D. y Niedderer, H. (eds.). Case-studies of the project «Labwork in Science Education». Working Paper 7.
KUHN, T. (1968). The essential tension. Chicago: University of Chicago Press.

JENKINS, E.W. (1979). From Armstrong to Nuffield. Londres: John Murria.

LEACH, J., MILLAR, R., RYDER, J. y SÉRÉ, M.G. (2000). Epistemological understanding in science learning: the consistency of representations across contexts. Learning and Instruction, 10(6), pp. 497-527.

LUNETTA, V.N. y TAMIR, P. (1979). Matching laboratory activities with teaching goals. The Science Teacher, 46(5), pp. 22-24.

N'TOMBELA, G.M. (1999). A marriage of inconvenience? School science practical work and the nature of science, en Leach, J. y Paulsen, A.C. (eds.). Practical work in science education: the face of science in schools, pp. 118-133. Roskilde: University of Roskilde Press.

PIAGET, J. (1972). Réussir et comprendre. París: Presses Universitaires de France.

RENAUD-COULON, A. y SÉRIEYX, H. (1999). Reprenons la Bastille. París: Éditions Village Mondial.

SÉRÉ, M.G. (1998). Improving science education: issues and research on innovative empirical and computer-based approches to labwork in Europe. Informe final del proyecto «Labwork in Science Education». Comisión Europea. http://formation.etud.u-psud.fr/didasco/index.htm

SÉRÉ, M.G., FERNÁNDEZ-GONZÁLEZ, M., LEACH, J., GONZÁLEZ-GARCÍA, F., DE MANUEL, E., GALLEGOS, A.J. y PERALES, F.J. (2001). Images of science linked to labwork: a survey of secondary school and university students. Research in Science Education.

SÉRÉ,M.G. y BENEY,M.(1998). Students' intellectual activities during stereotypical labwork at Undergraduate level, en Psillos, D. y Niedderer, H. (eds.). Teaching and learning in the science laboratory. Dordrecht: Kluwer.

SÉRÉ, M.G. (1992). Le déterminisme et le hasard dans la tête des élèves. Ou: de l'utilité du traitement statistique des mesures. Bulletin de l'Union des Physiciens, 740, pp. 87-96.

WAINMAIER, C. y SALINAS, J. (2001). Dimensiones de un aprendizaje comprensivo de las ciencias: las concepciones epistemológicas, en Retos de la enseñanza de las ciencias en el siglo XXI. VI Congreso internacional sobre Investigación en la Didáctica de las Ciencias. Barcelona.

WOOLNOUGH, B.E y ALLSOP, T. (1985). Practical science. Cambridge: Cambridge University Press. 


\section{ANEXO}

Se propuso un cuestionario, formado por cinco preguntas, a estudiantes de bachillerato y primer curso de universidad, en España (89 estudiantes de bachillerato y 37 de universidad) y en Francia (76 y 136). Dos de las preguntas se presentan aquí abajo:

\section{Cuando no se obtiene el mismo resultado de una medida a otra, ¿qué conclusión sacar?}

1.1. Se ha dado a dos grupos de alumnos un decilitro de aceite de oliva y se les pide medir su masa. Cada grupo hace cinco medidas.

Los resultados obtenidos, clasificados en orden creciente, son:

Medidas en gramos:

$\begin{array}{lrlllrl}\text { Grupo A } & 94,9 & 96,6 & 97,1 & 98,1 & 98,9 & \text { (media 97,12) } \\ \text { Grupo B } & 91,9 & 96,5 & 97,5 & 97,5 & 102,2 & \text { (media 97,12) }\end{array}$

¿Cuál de las siguientes afirmaciones te satisface más? (Señala con un aspa.)

a) Las dos series de medidas son igualmente buenas porque dan el mismo resultado.

b) Los resultados del grupo A son mejores porque la diferencia entre la medida más grande y la más pequeña es menor.

c) Los resultados del grupo B son mejores porque las medidas cubren un abanico más amplio de valores.

d) A partir de estos datos es imposible sacar ninguna conclusión referente a la calidad de las medidas.

Razona tu elección.

1.2. Se ha dado a otros dos grupos un decilitro de aceite de nuez y se les pide medir su masa. Cada grupo hace cinco medidas. Los resultados obtenidos, clasificados en orden creciente son:

Medidas en gramos:

$\begin{array}{lllllll}\text { Grupo A } & 92,4 & 93,3 & 93,4 & 94,0 & 94,4 & \text { (media 93,5) } \\ \text { Grupo B } & 91,9 & 93,3 & 94,9 & 95,0 & 96,9 & \text { (media 94,4) }\end{array}$

¿Cuál de las siguientes afirmaciones te satisface más? (Señala con un aspa.)

a) Como se tienen dos series de medidas, podría obtenerse un valor cogiendo (alguno/algunos/todos) datos de las dos.

b) Los resultados del grupo A son mejores porque la diferencia entre la medida más grande y la más pequeña es menor.

c) Los resultados del grupo B son mejores porque las medidas cubren un abanico más amplio de valores.

d) A partir de estos datos es imposible sacar ninguna conclusión referente a la calidad de las medidas.

Razona tu elección:

Si has elegido $a$, indica qué valor pondrías:

Si has elegido $d$, indica qué informaciones suplementarias necesitarías para poder determinar qué grupo es el mejor: 


\section{INVESTIGACIÓN DIDÁCTICA}

1.3. Un grupo de alumnos debe estudiar las propiedades de dos aceites vegetales diferentes. Toman un decilitro de cada uno y hacen cinco medidas de la masa en cada caso. Los resultados obtenidos son:

Medidas en gramos:

$\begin{array}{lllllll}\text { Aceite X } & 92,2 & 92,6 & 93,2 & 94,1 & 94,4 & \text { (media 93,3) } \\ \text { Aceite Y } & 93,6 & 94,1 & 94,3 & 95,0 & 95,4 & \text { (media 94,5) }\end{array}$

A partir de estos resultados, la conclusión es que «la densidad del aceite Y es mayor que la del aceite X».

¿Estás de acuerdo con esta conclusión? (Señala con un aspa.)

Estoy de acuerdo $\quad$ No estoy de acuerdo $\quad \square \quad$ No estoy seguro

Justifica tu respuesta:

\section{Cuando la observación no confirma lo que se espera:}

Se trata de una sesión de prácticas efectuada en el laboratorio por una clase. Los alumnos estudian muestras de hojas provenientes de plantas que han sido conservadas durante varios días en un armario oscuro y, por otra parte, hojas de plantas que han estado expuestas a la luz. El profesor sabe que debe haber almidón en estas últimas (las expuestas a la luz) y no en las primeras (las que quedaron en la oscuridad). Pero, por el momento, los alumnos no conocen lo que pueden encontrar.

Algunos grupos de alumnos de esta clase obtienen efectivamente el resultado esperado. Pero, para otros grupos, los tests del almidón no son nunca positivos.

A otros grupos, por el contrario, les sale positivo el test del almidón para algunas hojas que han estado a la luz (pero no para todas), y también para algunas hojas que han estado en la oscuridad (pero no para todas).

Estos resultados podrían deberse a que:

(Señalar con números las causas que creas más probables [1 para la más probable, 2 para la que le sigue en probabilidad, etc.]. Deja vacías las casillas de las que creas que no intervienen.)

- Algunos grupos se han equivocado en los tests.

- En biología, los fenómenos no siempre responden a las teorías.

- Deberían haber hecho varias experiencias de comprobación.

- Interviene algún otro factor (el origen de las hojas, el tiempo que han estado guardadas en la oscuridad, etc.).

- El test que se empleó para el almidón no es muy seguro.

- Otra causa (especificar).

Justifica tu respuesta. 